\title{
Tabela de esperança de vida e de fertilidade de Sipha flava (Forbes) (Hemiptera, Aphididae) alimentado com capim-elefante em diferentes temperaturas
}

\author{
Simone Alves de Oliveira ${ }^{1}$, Alexander Machado Auad ${ }^{2}$, Brígida Souza $^{3}$, Lívia Senra Souza $^{4}$, \\ Rodrigo Lopes Amaral ${ }^{5} \&$ Daniela Maria Silva ${ }^{6}$
}

\begin{abstract}
${ }^{1}$ Universidade Federal de Lavras, Lavras-MG. Brasil. sibiojf@yahoo.com.br.
${ }^{2}$ Pesquisador Embrapa Gado de Leite. amauad@cnpgl.embrapa.br.

${ }^{3}$ Departamento de Entomologia, Universidade Federal de Lavras, Lavras-MG, Brasil. brgsouza@ufla.br.

${ }^{4}$ Universidade Presidente Antônio Carlos, Juiz de Fora -MG, Brasil. liviabbsenra@ig.com.br.

${ }^{5}$ Universidade Federal de Juiz de Fora, Juiz de Fora-MG, Brasil. didolamaral@gmail.com.br.

${ }^{6}$ Centro de Ensino Superior de Juiz de Fora, Juiz de Fora-MG, Brasil. dandams_moni@yahoo.com.br.
\end{abstract}

\begin{abstract}
Life expectancy and fertility tables of Sipha flava (Forbes) (Hemiptera, Aphididae) fed elephant grass at different temperatures. The objective of this study was to estimate the life expectancy and fertility tables of Sipha flava (Forbes, 1884) fed with elephant grass at different temperatures. The investigations were conducted in climate controlled chambers at $12,16,20,24,28$ and $32^{\circ} \mathrm{C}$, UR $70 \pm 10 \%$ and 12 -hour photophase, with 150 insects at each temperature. There was gradual mortality at all temperatures studied. The longest life expectancy and longevity values for the aphids were at $12^{\circ} \mathrm{C}$. The highest net reproduction rates $\left(R_{0}\right)$ and longest population doubling time (DT) were also found at $12^{\circ} \mathrm{C}$. The survival $\left(1_{x}\right)$ started to diminish after 3.5 days at $24^{\circ} \mathrm{C}$ and after the first day at the other temperatures, gradually declining with the development of the insect. The time interval between each generation $(\mathrm{T})$ decreased as the temperature increased up to $28^{\circ} \mathrm{C}$. The highest specific fertility $\left(\mathrm{m}_{\mathrm{x}}\right)$ and total fecundity were recorded at $24^{\circ} \mathrm{C}$. The smallest value for innate capacity to increase in number $\left(\mathrm{r}_{\mathrm{m}}\right)$ were at $12^{\circ} \mathrm{C}$. The finite increase ratio $(\lambda)$ were highest at 20 e $24^{\circ} \mathrm{C}$. The temperature of $24^{\circ} \mathrm{C}$ is most suitable for the population density of $S$. flava to increase on elephant grass because it provides the highest values for the reproductive parameters.
\end{abstract}

KEYWORDS. Aphid; forage plant; reproductive capacity; temperature conditions.

RESUMO. Tabela de esperança de vida e de fertilidade de Sipha flava (Forbes) (Hemíptera, Aphididae) alimentado com capim-elefante em diferentes temperaturas. Objetivou-se estimar as tabelas de esperança de vida e de fertilidade para Sipha flava (Forbes, 1884) alimentados com capim-elefante, em diferentes temperaturas. Foram utilizadas câmaras climatizadas a $12,16,20,24,28$ e $32^{\circ} \mathrm{C}$, UR $70 \pm 10 \%$ e fotofase de 12 horas, contendo 150 insetos em cada condição térmica. Foi constatada mortalidade gradual em todas as temperaturas. A maior longevidade e maior esperança de vida dos afídeos foi a $12^{\circ} \mathrm{C}$. As taxas líquidas de reprodução ( $\mathrm{R}_{\mathrm{o}}$ e o tempo necessário para a população duplicar em número (TD) também foram maiores a $12^{\circ} \mathrm{C}$. A sobrevivência $\left(1_{x}\right)$ começou a diminuir a partir de 3,5 dias a $24^{\circ} \mathrm{C}$ e a partir do primeiro dia nas demais temperaturas, seguindo uma redução gradativa com o desenvolvimento do inseto. O intervalo de tempo entre cada geração (T) diminuiu com o aumento da temperatura, até $28^{\circ} \mathrm{C}$. A maior fertilidade específica $\left(\mathrm{m}_{\mathrm{x}}\right)$ e a maior fecundidade total média ocorreram a $24^{\circ} \mathrm{C}$. A capacidade inata de aumentar em número $\left(\mathrm{r}_{\mathrm{m}}\right)$ foi menor a $12^{\circ} \mathrm{C}$. A razão finita de aumento $(\lambda)$ foi maior a 20 e $24^{\circ} \mathrm{C}$. A temperatura de $24^{\circ} \mathrm{C}$ mostrou-se mais adequada para o desenvolvimento de $S$. flava em capim-elefante por proporcionar maiores valores para os parâmetros reprodutivos.

PALAVRAS-CHAVE. Afídeo; capacidade reprodutiva; condições térmicas; forrageira.

Os pulgões são considerados, devido suas características biológicas e impacto econômico nos cultivos agrícolas, como um dos grupos de insetos mais importantes em todo o mundo (Holman 1974). Dentre as espécies relatadas como pragas, Sipha flava (Forbes, 1884), conhecida como "pulgão amarelo", tem despertado a atenção dos pesquisadores. Esses afídeos encontram-se amplamente distribuídos (Blackman \& Eastop 2000) e estão associados a vários tipos de cereais (Blackman $\&$ Eastop 1984), podendo causar danos em muitas culturas, tais como cana-de-açúcar, trigo, cevada, centeio e gramíneas forrageiras (Kindler \& Dalrymple 1999; Blackman \& Eastop 2000; Miyasaka et al. 2007).
Dentre as gramíneas forrageiras, aquelas do gênero Penisetum se mostraram suscetíveis ao ataque de $S$. flava (Fukumoto \& Lee 2003). A espécie Penisetum purpureum (Schumach), conhecida como capim-elefante, é uma das mais utilizadas como alimento para a produção de leite no Brasil Central, destacando-se pela alta produtividade e qualidade da forragem (Xavier et al. 2001). É uma espécie tipicamente tropical (Dall'Agnol et al. 2004) e, devido ao fácil cultivo, elevada produção de forragem com bom valor nutritivo, boa palatabilidade, entre outras características, tem sido amplamente utilizada na dieta de bovinos (Costa \& Gonçalves 1988).

Estudos referentes ao crescimento populacional dos 
insetos são importantes para o entendimento da sua dinâmica (Harcourt 1969; Parra 1985) podendo ser usados como um indicador na determinação de estratégias de controle (Rabb et al. 1984; Nascimento et al. 1998). Trabalhos referentes às análises de tabela de vida visam abordar o desenvolvimento e sobrevivência de insetos, sendo a temperatura um dos principais fatores que influenciam esses parâmetros (Silveira Neto et al. 1976; Dixon 1998). Neste contexto, a determinação de tabelas de vida, submetendo os pulgões a várias temperaturas, são fundamentais para o entendimento da dinâmica populacional desses insetos (Hutchison \& Hogg 1984).

A qualidade da planta hospedeira na qual o inseto herbívoro se alimenta é fundamental para a determinação de diversos aspectos biológicos do seu desenvolvimento (Awmack \& Leather 2002) e, variações na fertilidade, longevidade e duração do período reprodutivo, podem determinar o surgimento de novos biótipos (Dixon 1990).

O afídeo, S. flava, é uma praga emergente em capim elefante, e estudos referentes a análises de tabelas de vida dessa espécie, nessa forrageira, são inexistentes, o que dificulta programas de manejo eficientes para o controle do inseto. Dessa forma, o objetivo deste trabalho foi determinar as tabelas de esperança de vida e de fertilidade para S. flava alimentado em capim-elefante, em diferentes temperaturas.

\section{MATERIALE MÉTODOS}

A pesquisa foi realizada no Laboratório de Entomologia da Embrapa, Gado de Leite em Juiz de Fora-MG. Inicialmente foi realizada a biologia do afídeo, sendo que os insetos foram mantidos em placas de plástico de $2,5 \mathrm{~cm}$ de diâmetro e $2,5 \mathrm{~cm}$ de altura, contendo uma camada de ágar de aproximadamente $1,0 \mathrm{~cm}$ de espessura. Em cada placa, sobre o Agar, foi disposto um disco foliar de $2 \mathrm{~cm}$ de diâmetro de capim elefante, cultivar Pioneiro, sobre o qual foi individualizada uma ninfa recémemergida do afídeo. As placas foram mantidas a 12, 16, 20, 24, 28 e $32^{\circ} \mathrm{C}$, utilizando-se 150 repetições, oriundos da criação em casa-de-vegetação, em cada condição térmica. Procederamse observações diárias, desde o nascimento até a morte do inseto. Com os dados da biologia foram elaboradas as tabelas de esperança de vida e de fertilidade.

Para a elaboração das tabelas de esperança de vida, determinaram-se o número de sobreviventes no início da idade $\mathrm{x}\left(\mathrm{l}_{\mathrm{x}}\right)$; número de indivíduos mortos durante o intervalo etário $\mathrm{x}\left(\mathrm{d}_{\mathrm{x}}\right)$; estrutura etária $\left(\mathrm{E}_{\mathrm{x}}\right)$, que corresponde ao número de indivíduos vivos entre um dia e outro; esperança de vida para indivíduos de idade $\mathrm{x}\left(\mathrm{e}_{\mathrm{x}}\right)$ e probabilidade de morte na idade $\mathrm{x}$ $\left(100 q_{x}\right)$, que indica a probabilidade de ocorrer morte dos indivíduos antes do tempo estabelecido em $\mathrm{e}_{\mathrm{x}}$. Utilizaram-se as seguintes equações conforme proposto por Silveira Neto et al. (1976): $\mathrm{E}_{\mathrm{x}}=\left[\mathrm{L}+\left(\mathrm{L}_{\mathrm{x}+1}\right)\right] / 2 ; \mathrm{e}_{\mathrm{x}}=\mathrm{T}_{\mathrm{x}} / \mathrm{L}_{\mathrm{x}}$ e $100 \mathrm{q}_{\mathrm{x}}=\left(\mathrm{d}_{\mathrm{x}} / \mathrm{l}_{\mathrm{x}}\right) \cdot 100$.

Para a tabela de vida de fertilidade, foram calculados as taxas de reprodução (Ro), intervalo de tempo entre as gerações (T), taxa intrínseca do aumento da população (rm), taxa finita de aumento $(\lambda)$, tempo necessário para a população duplicar em número de indivíduos (TD), médias do intervalo de idades $(x)$, fertilidade específica $\left(m_{x}\right)$ e probabilidade de sobrevivência $\left(1_{x}\right)$, aplicando-se as seguintes equações: $\operatorname{Ro}=\Sigma\left(m_{x} 1_{x}\right) ; T=\Sigma$ $\left(\mathrm{m}_{\mathrm{x}} \cdot 1_{\mathrm{x}} \cdot \mathrm{x}\right) / \Sigma\left(\mathrm{m}_{\mathrm{x} x} 1_{\mathrm{x}}\right) ; \mathrm{rm}=\log _{\mathrm{e}} \mathrm{Ro} / \mathrm{T}=\mathrm{In} \mathrm{Ro} / \mathrm{T} ; \lambda=\mathrm{e}^{\mathrm{rm}} \mathrm{e} \mathrm{TD}=\operatorname{In}(2) /$ $\mathrm{r}_{\mathrm{m}}$.

\section{RESULTADOS EDISCUSSÃO}

Tabela de esperança de vida.

A maior longevidade média dos afídeos ocorreu a $12^{\circ} \mathrm{C}$ (45,64 dias), seguida daquelas obtidas a $16,20,24,28$ e $32^{\circ} \mathrm{C}$ que corresponderam a 26,$6 ; 23,44 ; 20,14 ; 10,53$ e 9,5 dias, respectivamente (Figuras 1 e 2). Esses resultados evidenciam reduções no ciclo de vida em função da elevação da temperatura, corroborando com registros obtidos por diversos autores para outras espécies de afídeos. Ricci \& Kahan (2005) estudando Sipha maydis (Passerini, 1860) em plantas de cevada, a $20^{\circ} \mathrm{C}$, observaram um ciclo de vida de 52,68 dias, aproximadamente o dobro do registrado no presente estudo para a mesma temperatura. Iversen \& Harding (2007) constataram longevidade próxima a 80 dias para Phyllaphis fagi (Linnaeus) (72-84 dias) mantidos nas temperaturas de 12 e $15^{\circ} \mathrm{C}$, sendo que a $25^{\circ} \mathrm{C}$ este valor foi de 29 dias. Satar et al. (2005) registraram para Brevicoryne brassicae (L., 1758), em couve, maior longevidade quando os afídeos foram submetidos a temperaturas mais baixas, sendo que variações entre 30/ $35^{\circ} \mathrm{C}$ foram letais para o período ninfal. Michelotto et al. (2005) verificaram para $M$. persicae, em berinjela, uma longevidade de 13 a 41 dias quando os afídeos foram criados a 25 e $15^{\circ} \mathrm{C}$, respectivamente.

Foi constatada mortalidade gradual, tanto na fase ninfal quanto na adulta em todas as temperaturas estudadas (Figuras 1 e 2). A esperança de vida variou de 34,92 a $21,67\left(12^{\circ} \mathrm{C}\right) ; 18,38$ a $10,86\left(16^{\circ} \mathrm{C}\right) ; 18,63$ a $10,56\left(20^{\circ} \mathrm{C}\right) ; 14,47$ a $9,3\left(24^{\circ} \mathrm{C}\right) ; 5,27$ a $1,32\left(28^{\circ} \mathrm{C}\right)$ e 6,17 a $4,06\left(32^{\circ} \mathrm{C}\right)$ dias para a fase ninfal. Para a fase adulta constataram-se variações de 21,06 a $0,5\left(12^{\circ} \mathrm{C}\right)$; 10,65 a $0,5\left(16^{\circ} \mathrm{C}\right) ; 10,93$ a $0,5\left(20^{\circ} \mathrm{C}\right) ; 8,9$ a $0,5\left(24^{\circ} \mathrm{C}\right) ; 0,83$ a 0,5 $\left(28^{\circ} \mathrm{C}\right)$ e 3,7 a $0,5\left(32^{\circ} \mathrm{C}\right)$ (Figuras 1 e 2$)$, ocorrendo uma queda acentuada do início até o final da fase imatura nas duas temperaturas mais baixas e nas duas mais altas.

A probabilidade de morte antes do prazo estabelecido (100 $\mathrm{q}_{\mathrm{x}}$ ) ocorreu ao longo de toda a fase ninfal e adulta, nas quatro temperaturas mais baixas, não sendo verificada nas duas mais elevadas. A $12^{\circ} \mathrm{C}$ foi registrada a maioria dos valores nulos para este parâmetro, sendo verificados no $5^{\circ}, 13^{\circ}, 16^{\circ}$ e $19^{\circ}$ dias da fase ninfal e no $26^{\circ}, 30^{\circ}$ e $34^{\circ}$ dias da fase adulta.

As maiores taxas de mortalidade $\left(\mathrm{d}_{\mathrm{x}}\right)$ para afideos criados a $12^{\circ} \mathrm{C}$, ocorreram aos 0,5 e 55,5 dias de vida, com 6 e 7 indivíduos mortos, ocasiões em que foram geradas probabilidades de morte $\left(100 \mathrm{q}_{\mathrm{x}}\right)$ de 4 e $50 \%$. Para a temperatura de $16^{\circ} \mathrm{C}$ os maiores valores de $d_{x}$ ocorreram aos 2,5 e 24,5 dias de vida, com 8 e 24 indivíduos mortos e $100 \mathrm{q}_{\mathrm{x}}$ de 5,88 e 35,29\%. Para $20^{\circ} \mathrm{C}$, foi aos 0,5 e 18,5 dias de vida, com 7 e 13 mortos e $100 q_{x}$ igual a 4,7 e $15,48 \%$. Para a temperatura de $24^{\circ} \mathrm{C}$, aos 3,5 e 18,5 dias, com 8 e 13 mortos e 5,93 e 21,3\% de probabilidade de morte. A $28^{\circ} \mathrm{C}$ os maiores valores de $d_{x}$ ocorreram aos 7,5 e 9,5 

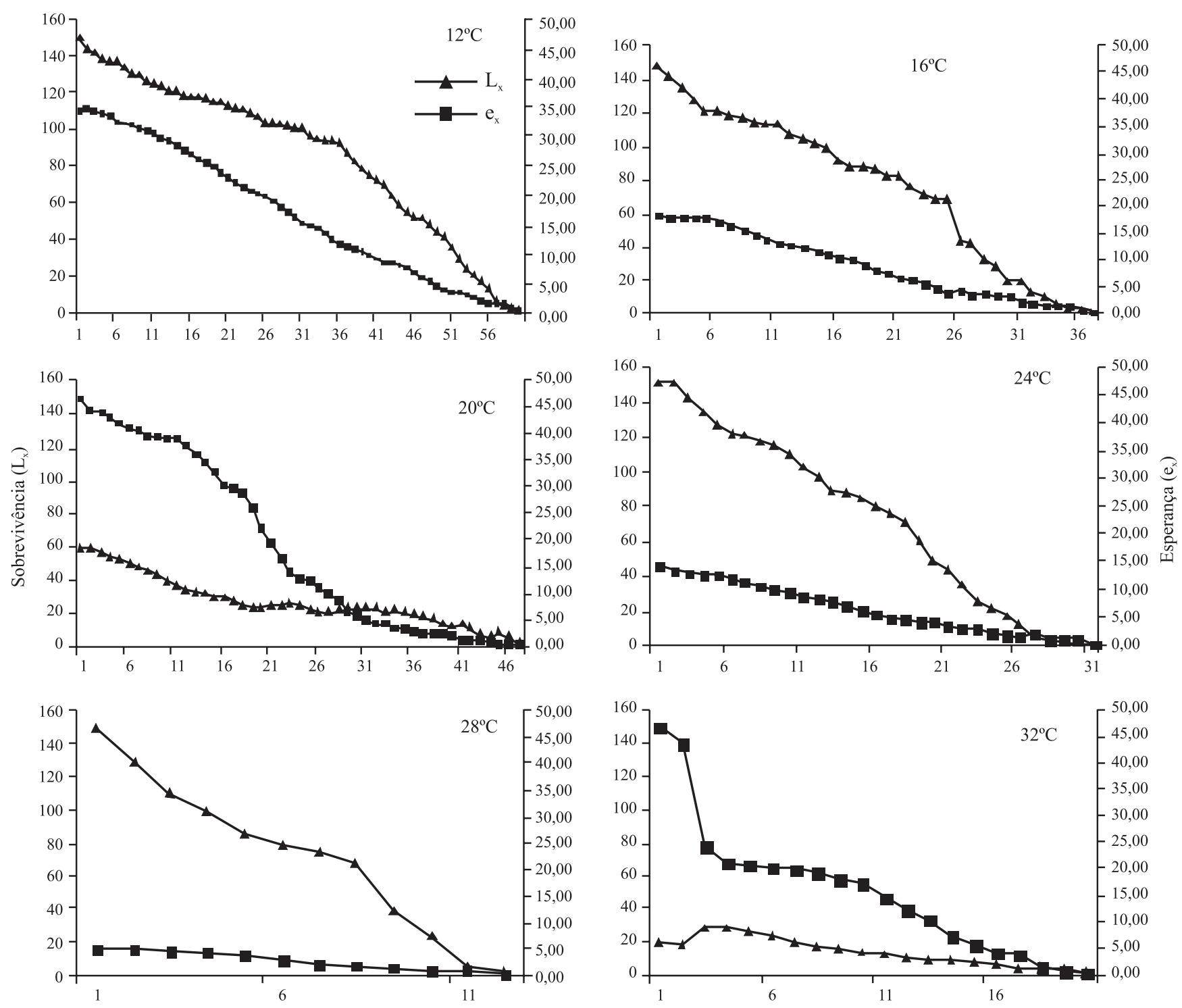

Fig. 1. Sobrevivência (Lx) e esperança de vida (ex) de Sipha flava em diferentes temperaturas, 12, 16, 20, 24,28 e $32^{\circ} \mathrm{C}, \mathrm{UR} 70 \pm 10 \%$ e fotofase de 12 horas. Juiz de Fora, MG-2007.

dias, com 29 e 18 mortos e probabilidade de morte igual a 42,65 e $75 \%$ e para a temperatura de $32^{\circ} \mathrm{C}$ aos 1,5 e 12,5 dias de vida, com 63 e 9 afideos mortos e uma probabilidade de morte de 45 e $27,27 \%$. Michelotto et al. (2005) registraram $64,29 \%$ de probabilidade de morte para $M$. persicae, à $25^{\circ} \mathrm{C}$, entre o $9^{\circ} \mathrm{e}$ $10^{\circ}$ dias, porcentagem cerca de três vezes maior que a obtida na temperatura mais próxima $\left(24^{\circ} \mathrm{C}\right)$ neste trabalho.

Os parâmetros analisados estão em desacordo com aqueles constatados na literatura para outras espécies de afídeos, o que é justificado, visto que, a esperança de vida do inseto esta associada à espécie em estudo (Tamaki et al. 1982) ou com à planta hospedeira utilizada como fonte de alimentação (Wale et al. 2000).

Com base na tabela de esperança de vida de S. flava este afídeo encontrará melhores condições para ocasionar injúrias e se tornar uma praga em potencial, em plantas de capim- elefante, nas regiões onde a temperatura média seja inferior à $24^{\circ} \mathrm{C}$.

Tabela de fertilidade de vida.

A sobrevivência $\left(1_{\mathrm{x}}\right)$ começou a diminuir a partir de 3,5 dias a $24^{\circ} \mathrm{C}$ e a partir do primeiro dia nas demais temperaturas, seguindo uma redução gradativa com o desenvolvimento do inseto (Figura 1). À $20^{\circ} \mathrm{Ce} 24^{\circ} \mathrm{C}$, a esperança de vida foi superior à sobrevivência, próximo ao $30^{\circ}$ dia de vida, denotando serem essas temperaturas favoráveis para o desenvolvimento dessa espécie, uma vez que, a sobrevivência desse inseto nestas temperaturas foi maior que o valor esperado.

O número médio de ninfas produzidas por fêmea de $S$. flava obtidas a 28 e $32^{\circ} \mathrm{C}$ foi de apenas 2,12 e 1 indivíduo, respectivamente (Figura 2), e o cálculo da tabela de vida de fertilidade acarretou valores negativos para a capacidade inata 

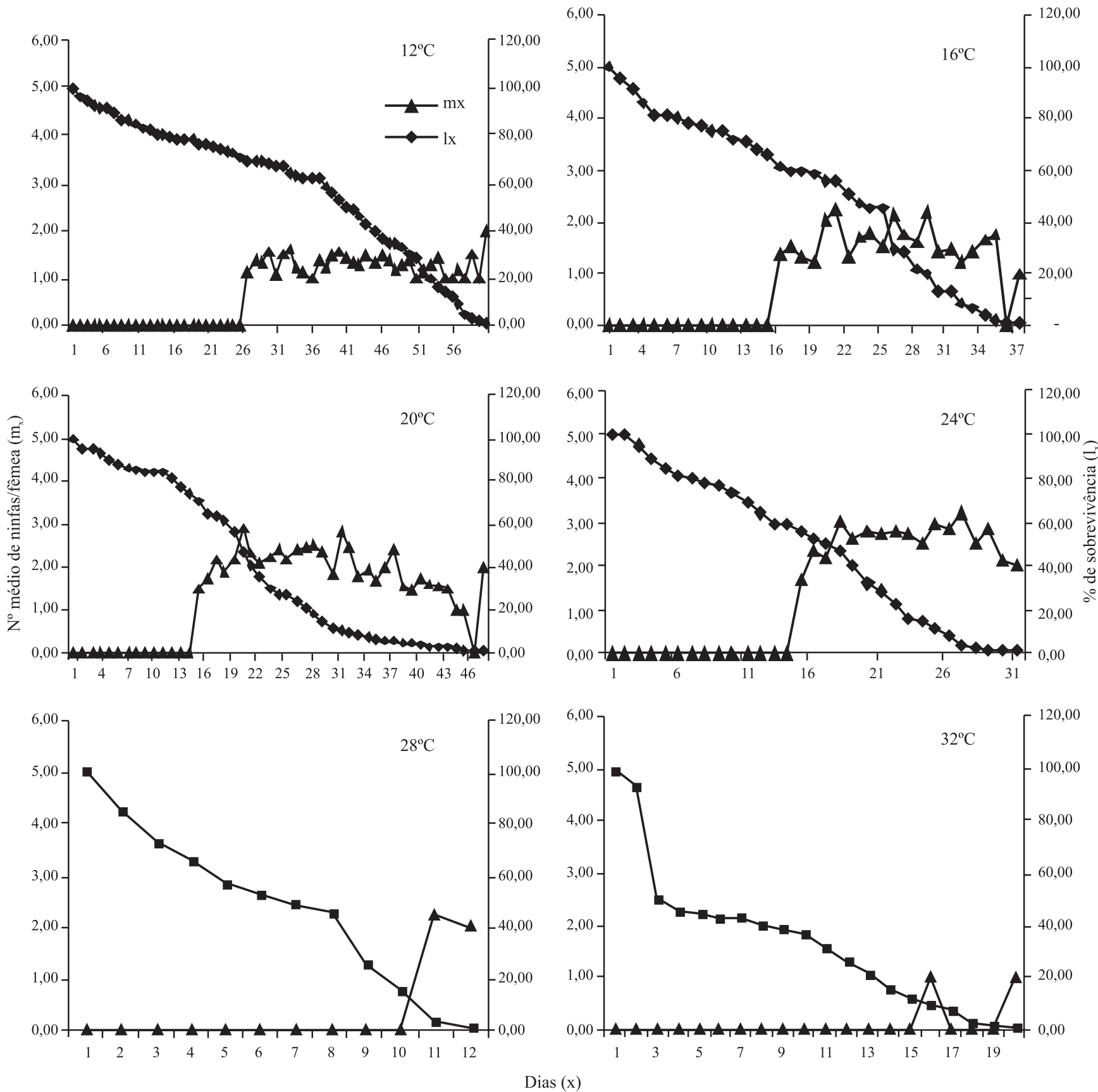

Fig. 2. Número médio de ninfas/fêmea $(\mathrm{mx})$ e porcentagem de sobrevivência (1x) de Sipha flava em diferentes temperaturas de 12, 16, 20, 24, 28 e $32^{\circ} \mathrm{C}$, UR $70 \pm 10 \%$ e fotofase de 12 horas. Juiz de Fora, MG -2007.

de aumentar em número (- 0,18 e - 0,14, respectivamente), denotando não serem tais temperaturas favoráveis para o processo reprodutivo dessa espécie de pulgão. Da mesma forma, McCornack et al. (2004) também registraram valor negativo para Aphis glycines Matsumura, a $35^{\circ} \mathrm{C}$, sendo sugerido que valores negativos desse parâmetro indicam uma redução no tamanho da população em conseqüência da alta mortalidade e da ausência de reprodução. Satar et al. (2005) registraram $100 \%$ de mortalidade das ninfas de $B$. brassicae quando criadas em temperaturas acima de $30^{\circ} \mathrm{C}$, condição em que não houve reprodução, sendo essa temperatura excluída das análises de tabela de vida. McCornack et al. (2004) estudando $A$. glycines a $20,25,30$ e $35^{\circ} \mathrm{C}$, verificaram que os adultos que sobreviveram à temperatura mais elevada não se reproduziram e todos morreram até o $11^{\circ}$ dia. Cividanes \& Souza (2003) registraram $100 \%$ de mortalidade das ninfas de $M$. persicae quando mantidas a $30^{\circ} \mathrm{C}$, sugerindo que essa espécie não é adaptada a elevadas temperaturas.

A maior fertilidade específica $\left(\mathrm{m}_{\mathrm{x}}\right)$ e a maior fecundidade total média para $S$. flava ocorreram a $24^{\circ} \mathrm{C}$, aos 26,5 dias do 
Tabela 1. Parâmetros de tabela de vida de fertilidade de Sipha flava em diferentes temperaturas de $12,16,20,24,28$ e $32^{\circ} \mathrm{C}$, UR $70 \pm 10 \%$ e fotofase de 12 horas. Juiz de Fora, MG - 2007.

\begin{tabular}{cccccc}
\hline Temperaturas $\left({ }^{\circ} \mathrm{C}\right)$ & \multicolumn{5}{c}{ Parâmetros } \\
\cline { 2 - 6 } & $\mathrm{T}$ & $\mathrm{R}_{0}$ & $\mathrm{r}_{\mathrm{m}}$ & $\lambda$ & $\mathrm{TD}$ \\
\hline 12 & 36,80 & 18,62 & 0,08 & 1,08 & 8,66 \\
16 & 21,90 & 10,92 & 0,11 & 1,11 & 6,30 \\
20 & 21,71 & 14,65 & 0,12 & 1,12 & 5,77 \\
24 & 18,78 & 9,71 & 0,12 & 1,12 & 5,77 \\
28 & 10,73 & 0,12 & $-0,18$ & 0,84 & 3,85 \\
32 & 15,77 & 0,10 & $-0,14$ & 0,87 & 4,95 \\
\hline
\end{tabular}

$\mathrm{T}=$ intervalo de tempo entre cada geração; $\mathrm{R}_{0}=$ taxa líquida de reprodução; $r_{m}=$ capacidade inata de aumentar em número; $\lambda=$ razão finita de aumento; $\mathrm{TD}=$ tempo necessário para população duplicar em número de indivíduos.

início do período reprodutivo, com 3,45 ninfas/fêmea/dia e um total de 24,88 ninfas/fêmea, evidenciando ser essa temperatura a mais adequada para a reprodução de S. flava (Figura 2). Resultado coincidente foi encontrado por Hentz \& Nuessly (2004), para a mesma espécie de afídeo, alimentado de sorgo, mantidos a $25^{\circ} \mathrm{C}$, com 3,3 ninfas/fêmea/dia; indicando que, nessa condição climática, não houve efeito da planta hospedeira nesses parâmetros biológicos do afídeo.

As taxas líquidas de reprodução $\left(\mathrm{R}_{0}\right)$ foram maiores nas temperaturas de $12^{\circ} \mathrm{Ce} 20^{\circ} \mathrm{C}$, sendo que os valores mais baixos foram obtidos nas temperaturas de $28^{\circ} \mathrm{C}$ e $32^{\circ} \mathrm{C}$ (Tabela 1). Trabalhando com M. persicae, Cividanes \& Souza (2003) e Satar et al. (2005) também registraram redução no valor de $\mathrm{R}_{0}$ com o incremento da temperatura. Hentz \& Nuessly (2004), estudando S. flava, e Barbosa (2004), estudando M. persicae, ambos à $25^{\circ} \mathrm{C}$, registraram $\mathrm{R}_{0}$ superiores aos constatados no presente estudo, com 36,6 e 67,3, respectivamente. Maiores valores para $\mathrm{R}_{0}$ também foram verificados por Bastos et al. (1996), para M. persicae, a $25^{\circ} \mathrm{C}$. Hayakawa et al. (1990) consideram que, devido à constatação de que adultos de afídeos apresentam alta taxa de mortalidade causada por fatores ambientais, é vantajoso para a espécie antecipar seu período reprodutivo.

O intervalo de tempo entre cada geração (T) diminuiu com o aumento da temperatura até $28^{\circ} \mathrm{C}$, sendo de 36,$8 ; 21,9 ; 21,71$; 18,78 e 10,73 dias a $12,16,20,24$ e $28^{\circ} \mathrm{C}$, respectivamente; porém à $32^{\circ} \mathrm{C}$ ocorreu um aumento, sendo de 15,77 (Tabela 1). Barbosa (2004), Auad e Moraes (2003) e Godoy \& Cividanes (2002) para diferentes espécies de afídeos, também, verificaram queda no tempo entre gerações com o aumento da temperatura.

Valores negativos para a capacidade inata de aumentar em número $\left(\mathrm{r}_{\mathrm{m}}\right)$ foram registrados para as temperaturas de $28^{\circ} \mathrm{C} \mathrm{e}$ $32^{\circ} \mathrm{C}$. A $20^{\circ} \mathrm{C}$ e $24^{\circ} \mathrm{C}$ foram obtidos os valores mais elevados de $\mathrm{r}_{\mathrm{m}}$, de 0,12 , em ambas as condições (Tabela 1), sugerindo ser estas temperaturas as mais favoráveis para o aumento da população dessa espécie de pulgão. Hentz e Nuessly (2004) encontraram, para esse afídeo alimentados de sorgo, a $25^{\circ} \mathrm{C}$, $r_{m}$ de 0,314 , valor cerca de duas vezes maior que o observado no presente trabalho; denotando efeito da planta hospedeira, da utilização de biótipos diferentes ou da associação destes.
A razão finita de aumento $(\lambda)$ foi maior nas temperaturas de 20 e $24^{\circ} \mathrm{C}$, correspondendo a 1,12 para ambas as condições (Tabela 1). Para M. persicae, Barbosa (2004) encontrou 1,16 e 1,36 à 15 e $25^{\circ} \mathrm{C}$, respectivamente, denotando uma semelhança com os valores encontrados no presente estudo. Razmjou et al. (2006) registraram valores de $\lambda$ entre 1,28 e 1,39 para $A$. gossypii criado em diferentes cultivares de algodão, a $27,5^{\circ} \mathrm{C}$. Bastos et al. (1996) ressaltaram que em condições de campo, vários fatores ecológicos desfavoráveis à multiplicação de afídeos podem reduzir o número de descendentes.

O tempo necessário para a população duplicar em número de indivíduos (TD) foi maior a $12^{\circ} \mathrm{C}(8,66)$, evidenciando que em temperaturas mais baixas, o tempo para a duplicação da densidade populacional de $S$. flava é maior (Tabela 1).

\section{CONCLUSÕES}

A maior longevidade, bem como a maior esperança de vida para $S$. flava ocorreu a $12^{\circ} \mathrm{C}$ e as maiores taxas de mortalidade foram observadas a $28^{\circ} \mathrm{C}$ e $32^{\circ} \mathrm{C}$.

A maior fertilidade específica e a maior fecundidade total média encontram-se a $24^{\circ} \mathrm{C}$, evidenciando ser esta temperatura a mais adequada, nas condições do ensaio, para o desenvolvimento de S. flava em capim-elefante, por proporcionar os maiores valores para os parâmetros reprodutivos da espécie.

\section{REFERÊNCIAS}

Auad, A. M. \& J. C. Moraes. 2003. Biological aspects and life table Uroleucon ambrosiae (Thomas, 1878) as a function of temperature. Scientia Agrícola 60: 657-662.

Awmack, C. S \& S. R. Leather. 2002. Host plant quality and fecundity in herbivorius insects. Annual Review of Entomology 7: 817844.

Barbosa, L. R. Aspectos biológicos de Myzus persicae (Sulzer, 1776) (Hemiptera: Aphididae) e interação com Chrysoperla externa (Hagen,1861) (Neuroptera: Chrysopidae) em plantas de pimentão. 2004. 113 p. Tese (Doutorado). Universidade Federal de Lavras.

Bastos, C. S.; M. C. Picanso; G. L. D. Leite \& J. M. Araújo. 1996. Tabelas de fertilidade e de esperança de vida de Mysus persicae (Sulzer) (Homoptera) em couve-comum. Científica 24: 187-197.

Blackman, R. L. \& V. F. Eastop. 2000. Aphids on the worlds crops: an identification and information guide, 2 nd ed., Chicherster, John Wiley \& Sons, $466 \mathrm{p}$.

Blackman, R. L. \& V. P. Eastop. 1984. Aphids on the World's Crops. An Identification Guide. New York, John Wiley \& Sons, p. 120134.

Cividanes, F. J. \& V. P. Souza. 2003. Exigências térmicas e Tabelas de Vida de fertilidade de Myzus persicae (Sulzer) (Hemiptera: Aphididae) em laboratório. Neotropical Entomology 32: 413-419.

Costa, N. DE L. \& C. A. Gonçalves. 1988. Épocas de vedação e utilização de capineiras de capim-elefante em Porto Velho, Rondônia. Pasturas Tropicales 10: 34-37.

Dall'Agnol, M.; Scheffer-Basso; \& J. A. L. Nascimento. 2004. Produção de forragem de capim-elefante sob clima frio. Curva de Crescimento e Valor Nutritivo. Revista Brasileira de Zootecnia 33: 1110 1117

Dixon, A. F. G. 1990. Ecological interations of aphids and their host plants. p. 7-19. In: Aphid plant genotype interations. R. K. 
Campbell \& R. D. Eikenbary (eds.). Amsterdam. Elsevier.

Dixon, A. F. G. 1998. Aphid ecology an optimization approach. 2. ed. London, Chapman and Hall, 300 p.

Fukumoto, G. K. \& C. N. Lee. 2003. Kikuyugrass for forage. Cooperative Extension Service. 116 p.

Godoy, K. B. \& F. J. Cividanes. 2002. Tabelas de fertilidade e esperança de vida para Lipaphis erisimi (Kalt.) (Hemiptera:Aphididae) sob condições de laboratório e campo. Neotropical Entomology 31: $41-48$.

Harcourt, D. G. 1969. The development and use of life tables in the study of natural insect populations. Annual Review Entomology 17: $175-196$.

Hayakawa, D. L.; E. Grafius \& F. W. Stehr. 1990. Effects of temperature on longevity, reproduction, and development of the asparagus aphid (Homoptera: Aphididae) and the parasitoid, Diaretiella rapae (Hymenoptera: Braconidae). Environmental Entomology 19: 890-897.

Hentz, M. \& G. Nuessly. 2004. Development, Longevity, and Fecundity of Sipha flava (Homoptera: Aphididae) Feeding on Sorghum bicolor. Environmental Entomology 33: 546-553.

Holman, J. 1974. Los afidos de Cuba. Instituto Cubano del Libro, La Habana. 304 p.

Hutchison, W. D. \& D. B. Hogg. 1984. Demographic statitics for the peã aphid (Homoptera: Aphididae) in Wisconsin and a comparison with other populations. Environmental Entomology 13: 11731181 .

Iversen, T. \& S. Harding. 2007. Life table parameters affecting the population development of the wooly beech aphid, Phyllaphis fagi. Entomologia Experimentalis et Applicata 123: 109-117.

Kindler, S. D. \& R. L. Dalrymple. 1999. Relative susceptibility of cereals and pasture grasses to the yellow sugarcane aphid (Homoptera: Aphididae). Journal of Agricultural and Urban Entomology 16: 113-122.

McCornack, B. P.; D. W. Ragsdale \& R. C. Venette. 2004. Demography of soybean aphid (Homoptera: Aphididae) at summer temperatures. Entomological Society of America 97: 854-861.

Michelotto, M. D.; N. R. Chagas Filho; R. A. da Silva \& A. C. Busoli. 2005. Life Tables of Myzus persicae (Sulzer, 1776) (Hemiptera: Aphididae) on eggplant at differents temperatures. Bioscience Journal 21: 41-46.
Miyasaka, S. C.; J. D. Hansen; T. G. Mcdonald \& G. K. Fukumoto. 2007. Effects of nitrogen and potassium in kikuyu grass on feeding by yellow sugarcane. Crop Protection 26: 511-517.

Nascimento, M. L.; D. F. Capalbo; G. J. Moraes; E. A. de Nardo; H. N. de Maia \& R. C. Oliveira. 1998. Effect of formulation of Bacillus thuringiensis Berliner var. Kurstaki on Podisus nigrispinus (Heteroptera: Pentatomidae: Asopinae). Journal Invertebrate Pathology 72: 178-180.

Parra, J. R. P. 1985. Biologia comparada de Perileucoptera coffeella (Guérin-Mèneville, 1842) (Lepdoptera: Lyonetiidae) visando ao seu zoneamento ecológico no estado de São Paulo. Revista Brasileira de Entomologia 29: 45-76.

Rabb, R. L.; G. R. Defoliari \& G. G. Kennedy. 1984. An ecological approach to managing insect populations. p. 697-728. In: C. B. Huffaker \& R. L. Rabb (eds.), Ecological Entomology, New York, John Wiley, 916 p.

Razmjou, J.; S. Moharramipour; Y. Fathipour \& S. Mirhoseini. 2006. Demographic parameters of cotton aphid, Aphis gossypii Glover (Homoptera: Aphididae) on five cotton cultivars. Insect Science 13: $205-210$.

Ricci. M. \& A. E. Kahan. 2005. Biological and populational aspect of Sipha maidis (Passerini) y Schizaphis graminum (Rondani) on barley. Revista de la Facultad de Ciencias Agrarias 2: 25-32.

Satar, S.; U. Kersting \& M. R. Ulusoy. 2005. Temperature dependent life history traits of Brevicoryne brassicae (L.) (Homoptera: Aphididae) on white cabbage. Turkish Journal of Agriculture and Forestry 29: 341-346.

Silveira Neto, S.; O. Nakano; D. Barbin \& N. A. Vilanova. 1976. Manual de ecologia dos insetos. São Paulo, Agronômica Ceres, 419 p.

Tamaki, G.; B. Annis; L. FOX; R. K. Gupta \& A. Meszleny. 1982. Comparison of yellow holocycliv and green anholocyclic strains of Myzus persicae (Sulzes): low temperature adaptability. Environmental Entomology 11: 231-233.

Wale, M.; J. Bekele \& S. Emiru. 2000. Biology of the pea aphid, Acyrthosiphon pisum (Harris) (Homoptera: Aphididae) on coolseason legumes. Journal of Insect Science and Its Application 3: $171-180$.

Xavier, D. F.; M. M. Carvalho; M. A. Botrel; V. P. Freitas \& R. S. Verneque. 2001. Efeito do manejo pós-plantio no estabelecimento de pastagem de Capim-elefante. Revista Brasileira de Zootecnia 30: $1200-1203$. 